\title{
The role of fortified foods and nutritional supplements in the diets of older Irish adults
}

\section{Abstract}

Low intakes and suboptimal status of vitamin $\mathrm{D}$, riboflavin, vitamin B12, folate and calcium have been reported in older adults across Europe. Dietary strategies to improve micronutrient intakes and status could include food fortification (mandatory or voluntary) and/ or the use of nutritional supplements. This study aims to examine the impact of fortified food consumption and nutritional supplement use on nutrient intakes and nutritional status of vitamin D, riboflavin, vitamin B12, dietary folate equivalents (DFE) and calcium in older Irish adults.

Analyses were based on the National Adult Nutrition Survey (NANS) (2008-2010). A 4 day semi-weighed food record was used to collect food and beverage intake data (including nutritional supplement use) from a nationally representative sample of 1500 adults (226 older adults $(\geq 65 \mathrm{y}))$. Blood samples $(n 1126(n 145 \geq 65 \mathrm{y}))$ were collected, processed and analysed using standard operating procedures. Nutrient intakes were estimated using WISP ${ }^{\mathcal{O}}$ based on UK and Irish food composition data. Fortified foods were identified as those that had one or more micronutrients added in the ingredient list and a nutritional supplement was defined as a supplement containing vitamins and/or minerals (i.e. excludes herbal/non-nutritional supplements). A fortified food consumer or nutritional supplement user was defined as a participant who consumed a fortified food or took a nutritional supplement at any time over the 4-day recording period. Usual intakes of micronutrients were calculated via the NCI-method using SAS ${ }^{\mathcal{O}}$ Enterprise Guide and analyses of biomarker data were carried out using SPSS $^{\odot}$.

The consumption of fortified foods and/or use of nutritional supplements increased mean intakes of vitamin D (3.6 to $6.9 \mu \mathrm{g} / \mathrm{d}$ ), riboflavin (1.6 to $2.3 \mathrm{mg} / \mathrm{d}$ ), vitamin B12 (4.5 to $6.0 \mu \mathrm{g} / \mathrm{d})$, DFE (228 to $408 \mu \mathrm{g} / \mathrm{d})$ and calcium $(784$ to $947 \mathrm{mg} / \mathrm{d})$ in older Irish adults and reduced the prevalence of inadequate intakes of these micronutrients by up to $40 \%$. Furthermore, consumers of fortified foods and/or nutritional supplements had improved biomarker status and reduced prevalence of low/deficient status for vitamin D (62 vs $16 \%$ ), riboflavin (65 vs $11 \%$ ), vitamin B12 (8 vs $0 \%$ ) and folate (serum folate: 18 vs $0 \%$; red blood cell folate: $0 \%$ across all groups) when compared to non-consumers.

This study has shown that fortified foods and/or nutritional supplements represent an opportunity to improve intakes and status of key micronutrients in older adults. The data presented in this study will serve to inform the development and implementation of updated dietary recommendations for older adults in Ireland.

\section{Conflict of Interest}

There is no conflict of interest 